\title{
PROPERTIES OF LOCALIZED PROTONS IN NEUTRON STAR MATTER AT FINITE TEMPERATURES*
}

\author{
Adam Szmagliński, Sebastian Kubis, Weodzimierz Wójcik \\ Institute of Physics, Cracow University of Technology \\ Podchorążych 1, 30-084 Kraków, Poland
}

(Received December 2, 2013)

\begin{abstract}
We study properties of the proton component of neutron star matter for realistic nuclear models. Vanishing of the nuclear symmetry energy implies proton-neutron separation in dense nuclear matter. Protons which form admixture tend to be localized in potential wells. Here, we extend the description of proton localization to finite temperatures. It appears that the protons are still localized at temperatures typical for hot neutron stars. That fact has important astrophysical consequences. Moreover, the temperature inclusion leads to unexpected results for the behavior of the proton localized state.
\end{abstract}

DOI:10.5506/APhysPolB.45.249

PACS numbers: 21.65.Cd, 26.60.-c, 65.90.+i, 97.60.Jd

\section{Introduction}

The structure of nuclear matter at high densities and high temperatures is still the interesting and unsolved problem. The behavior of matter during supernova explosions and neutron stars collisions concerns nonzero temperature case of neutron stars. The asymmetric nuclear matter at finite temperatures in the Thomas-Fermi model was studied in [1]. The behavior of proton component in strongly asymmetric nuclear matter was discussed in $[2,3]$ and developed with more advanced calculations of the neutron background distribution around the single proton $[4,6]$. In this paper, we extend the proton localization problem to finite temperatures, of the order of $10 \mathrm{MeV}$, typical for condition occurring in the supernova explosions and neutron star merging.

* Presented at the XXXIII Mazurian Lakes Conference on Physics, Piaski, Poland, September 1-7, 2013. 


\section{Proton localization at zero temperature}

In our previous calculations, a specific family of realistic nuclear models was applied $[7,8]$. For this family, the nuclear symmetry energy vanishes at some density above saturation density, $n_{0}$. According to recent analysis [5], the symmetry energy slope at $n_{0}$ is rather small $40<L<60 \mathrm{MeV}$ and such value does not contradict that $E_{\mathrm{s}}$ may cease to increase and take very low values at higher densities. Such a form of the symmetry energy makes very low proton abundance at high densities (about a few percent) and leads to the proton-neutron instability [9]. The instability allows for the existence of the ferromagnetic state in neutron star core. This kind of ferromagnetic state stands for possible explanation for observed long-lived magnetic field in neutron star. In this way, being hypothetical, the specific form of the $E_{\mathrm{s}}$ presents an interesting "parameter study".

In pure neutron matter at higher densities, the proton chemical potential is a growing function of baryon number density. When protons constitute a small admixture to neutrons, the energy of the whole system can be lowered by creation of the neutron bubble around a single proton. Protons tend to be localized in potential wells corresponding to the neutron matter inhomogeneities induced by the protons in the neutron medium.

The energetically favorable ground state of matter was found by comparing the energy of a normal phase with uniform density and a phase with localized protons. The total volume of the system was divided into WignerSeitz cells, each of them enclosing a single localized proton [2, 3]. The localized proton was described by the Gaussian wave function with the variational parameter root mean square (r.m.s.) radius $R_{P}$ and the neutron background was treated in the Thomas-Fermi approximation.

\section{Properties of nuclear matter at $T>0$}

At finite temperatures, the ground state of nuclear matter is found by minimizing the free energy of the Wigner-Seitz cell. In order to solve the problem, we used the standard formulae which are shown below. The free energy per baryon is

$$
F=\left(\varepsilon\left(n_{N}, n_{P}, T\right)-T\left(n_{N} S_{N}+n_{P} S_{P}\right)\right) / n .
$$

Now, the energy density $\varepsilon\left(n_{N}, n_{P}, T\right)$ depends on baryon number densities of neutrons $n_{N}$ and protons $n_{P}\left(n_{N}+n_{P}=n\right)$, and on the temperature as well. The entropy per baryon is

$$
S_{N, P}=\frac{5}{3} \frac{1}{n_{N, P}} \frac{1}{4 \pi^{2}}\left(2 m_{N, P}^{*} T\right)^{3 / 2} J_{3 / 2}\left(\eta_{N, P}\right)-\frac{1}{2} \eta_{N, P},
$$


where the effective nucleon masses are denoted by $m_{N, P}^{*}$. The unknown quantity $\eta_{N, P}$ is eliminated from the relation

$$
n_{N, P}=\frac{2}{(2 \pi)^{2}}\left(2 m_{N, P}^{*} T\right)^{3 / 2} J_{1 / 2}\left(\eta_{N, P}\right) .
$$

Appearing above Fermi integrals are defined as follows

$$
J_{\nu}(\eta)=\int_{0}^{\infty} d x \frac{x^{\nu}}{1+e^{x-\eta}} .
$$

Thermodynamic identities allow to express the nucleon chemical potentials as the derivatives of the free energy per baryon (here $f$ is the free energy density)

$$
\mu_{N, P}=\frac{\partial f}{\partial n_{N, P}}=n \frac{\partial F}{\partial n_{N, P}}+F .
$$

We calculate the free energy difference between the localized state of protons and the state with uniform matter $\Delta F=F_{L}-F_{0}$ for the FriedmanPandharipande-Ravenhall (FPR) model. This difference $\Delta F$ as the function of the average neutron number density is shown in the left panel of Fig. 1 for various temperatures $T=0,20,50 \mathrm{MeV}$. The negative values of the $\Delta F$ at some density indicates the ground state of matter with localized protons. Zero value corresponds to the threshold density $n_{\text {loc }}$ above which the localization of protons takes place. The dependence of the threshold density on the temperature is plotted in the right panel of Fig. 1. The results of our calculations show that the threshold density for the localization $n_{\text {loc }}$
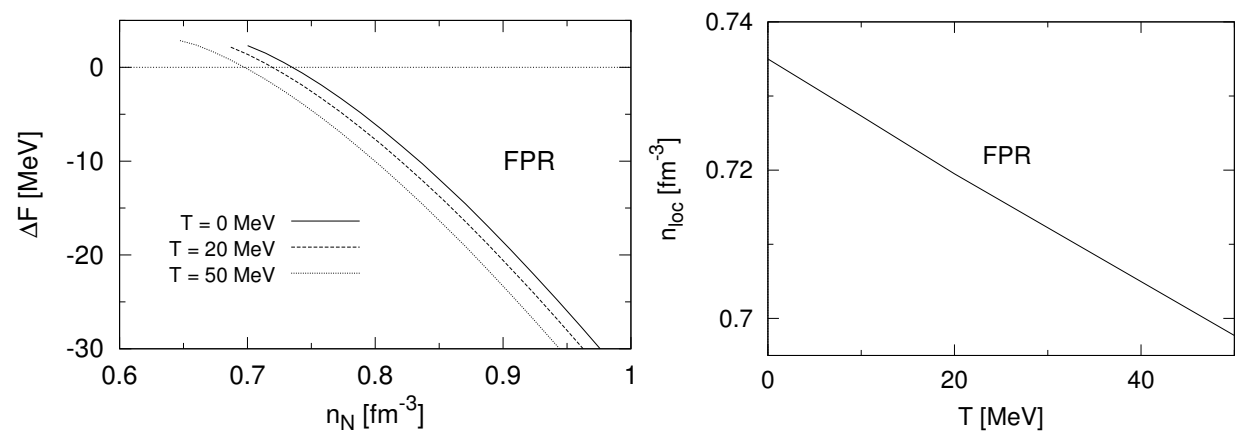

Fig. 1. Left: the free energy difference $\Delta F$ at the minimum as the function of density at different temperatures for the FPR model. Right: the threshold density $n_{\text {loc }}$ above which the localization is predicted as a function of the temperature of neutron matter. 
decreases with the increasing temperature. This means that the localization is amplified by the temperature. An interesting result was obtained for the r.m.s. radius of the proton wave function $R_{P}$, Fig. 2, and for the distribution of neutron density at different temperatures, Fig. 3. Namely, when the temperature increases, the proton wave function is more squeezed by the surrounding neutrons. This is unexpected result as one may suspect that the temperature would spread out the wave function rather than compress it. We have also performed the same calculations for other realistic nuclear models and obtained similar results as above.

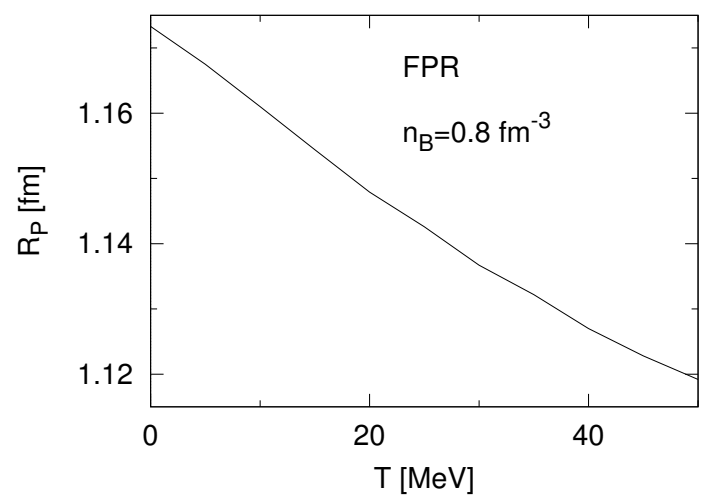

Fig. 2. The r.m.s. radius of proton wave function at fixed density $n_{B}=0.8 \mathrm{fm}^{-3}$ as a function of the temperature for the FPR model.

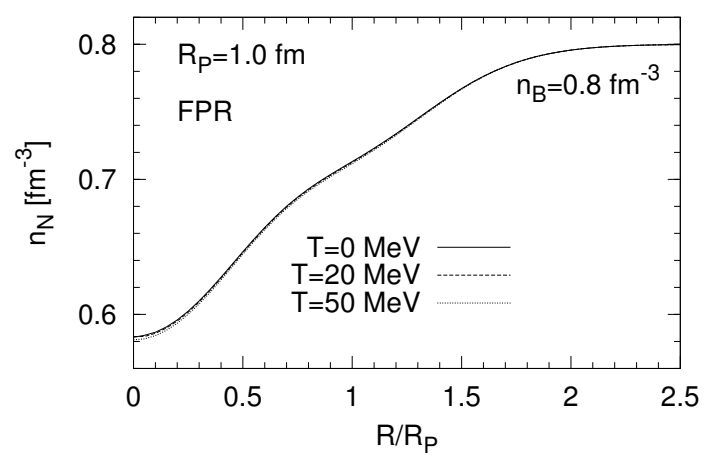

Fig. 3. The neutron density distribution at different temperatures.

\section{Conclusions}

In summary, we want to emphasize that the inclusion of the temperature in the proton localization phenomenon results in important consequences for neutron stars. First, the temperature inclusion leads to the counter-intuitive 
effects. It lowers the localization threshold density and diminishes the size of the proton wave function. Second, the localization is still present even in the case of a very high temperature like $50 \mathrm{MeV}$. The localized protons could be responsible for the ferromagnetic properties of the inner core [10]. Our calculations suggests that the ferromagnetic core is formed very early during the protoneutron star formation or, from the other side, survive the merging of neutron star binary when the matter is reheated to a very high temperature.

\section{REFERENCES}

[1] K. Strobel, F. Weber, M.K. Weigel, Z. Naturforsch. 54a, 83 (1999).

[2] M. Kutschera, W. Wójcik, Phys. Lett. B223, 11 (1989).

[3] M. Kutschera, W. Wójcik, Phys. Rev. C47, 1077 (1993).

[4] M. Kutschera, S. Stachniewicz, A. Szmagliński, W. Wójcik, Acta Phys. Pol. B 33, 743 (2002).

[5] J.M. Lattimer, Y. Lim, Astrophys. J. 771, 51 (2013).

[6] A. Szmagliński, W. Wójcik, M. Kutschera, Acta Phys. Pol. B 37, 277 (2006).

[7] R.W. Wiringa, V. Fiks, A. Fabrocini, Phys. Rev. C38, 1010 (1988).

[8] V.R. Pandharipande, V.K. Garde, Phys. Lett. B39, 608 (1972).

[9] M. Kutschera, Phys. Lett. B340, 1 (1994).

[10] M. Kutschera, W. Wójcik, J. Mag. Mag. Mat. 140-144, 2183 (1995). 